University of Nebraska - Lincoln

DigitalCommons@University of Nebraska - Lincoln

\title{
Captive Coyotes Compared to Their Counterparts in the Wild: Does Environmental Enrichment Help?
}

\author{
John A. Shivik \\ USDA Wildlife Services National Wildlife Research Center; Logan, Utah. \\ Gernrna L. Palmer \\ University of Exeter \\ Eric M. Gese \\ USDA/APHIS/WS National Wildlife Research Center, eric.gese@usu.edu \\ Britta Osthaus \\ University of Exeter
}

Follow this and additional works at: https://digitalcommons.unl.edu/icwdm_usdanwrc

Part of the Environmental Sciences Commons

Shivik, John A.; Palmer, Gernrna L.; Gese, Eric M.; and Osthaus, Britta, "Captive Coyotes Compared to Their Counterparts in the Wild: Does Environmental Enrichment Help?" (2009). USDA National Wildlife Research Center - Staff Publications. 969.

https://digitalcommons.unl.edu/icwdm_usdanwrc/969

This Article is brought to you for free and open access by the U.S. Department of Agriculture: Animal and Plant Health Inspection Service at DigitalCommons@University of Nebraska - Lincoln. It has been accepted for inclusion in USDA National Wildlife Research Center - Staff Publications by an authorized administrator of DigitalCommons@University of Nebraska - Lincoln. 


\title{
Captive Coyotes Compared to Their Counterparts in the Wild: Does Environmental Enrichment Help?
}

\author{
John A. Shivik, ${ }^{1}$ Gemma L. Palmer, ${ }^{2}$ Eric M. Gese, ${ }^{1}$ \\ and Britta Osthaus ${ }^{2}$ \\ ${ }^{1}$ USDA Wildlife Services National Wildlife Research Center; Logan, Utah \\ ${ }^{2}$ School of Psychology, University of Exeter, Exeter, United Kingdom
}

This article attempts to determine the effects of environment (captive or wild) and a simple form of environmental enrichment on the behavior and physiology of a nonhuman animal. Specifically, analyses first compared behavioral budgets and stereotypic behavior of captive coyotes (Canis latrans) in kennels and pens to their counterparts in the wild. Second, experiments examined the effect of a simple form of environmental enrichment for captive coyotes (food-filled bones) on behavioral budgets, stereotypies, and corticosteroid levels. Overall, behavioral budgets of captive coyotes in both kennels and pens were similar to those observed in the wild, but coyotes in captivity exhibited significantly more stereotypic behavior. Intermittently providing a bone generally lowered resting and increased foraging behaviors but did not significantly reduce stereotypic behavior or alter corticosteroid levels. Thus, coyote behavior in captivity can be similar to that exhibited in the wild; in addition, although enrichment can affect proportions of elicited behaviors, abnormal behaviors and corticosteroid levels may require more than a simple form of environmental entichment for their reduction.

In the wild, behaviors such as searching, processing, and eating are important components of a nonhuman animal's natural activity budget (Herbers, 1981). Environmental conditions can influence the success of foraging, which in turn can

This article not subject to United States copyright law.

Correspondence should be sent to John A. Shivik, Wildlife Services National Wildlife Research Center, Department of Wildland Resources, Utah. State University, 163 BNR Building, Logan, UT 84322-5295. Email: john.shivik@aphis.usda.gov 
influence how animals partition their foraging versus other behaviors (Carlstead, Seidensticker, \& Baldwin, 1991). Feeding is a particularly important aspect of a carnivore's daily activity budget, and fundamental aspects of food acquisition behavior include the ability to search, locate, and capture food (Shepherdson, Carlstead, Mellen, \& Sidensticker, 1993). In a captive environment where food is presented at regular times in one position, animals have little motivation or opportunity to forage (Newberry, 1995). It is recognized that stereotypic behaviors (repetitive movements with no apparent goal or function) can be influenced by the timing and manner of food presentation (Carlstead et al., 1991; Garner, Mason, \& Smith, 2003; Terlouw, Lawrence, \& Illius, 1991) and that frustrating efforts to access food can result in stress (Dantzer, 1986).

The behaviors and activity budgets of captive animals should ideally be similar, if not identical, to those of conspecifics in the wild (Renner \& Lussier, 2002; Ŝpinka, 2006), although there is some contention that similarity to wild behavior provides an adequate metric for welfare (Veasey, Waran, \& Young, 1996). In research facilities, however, if animals demonstrate natural behaviors within their confined living conditions, experimental results may have stronger inference from captive to wild situations.

Although corticosteroid levels can have a variety of interpretations, elevated corticosteroid levels are also considered indicators of stress for wild and domestic animals (Becker et al., 1985, Morton, Anderson, Foggin, Kock, \& Tiran, 1995). Thus, evidence of improvements (or declines) in animal welfare may also be evident in corticosteroid levels. Monitoring corticosteroids under different husbandry regimes may be useful for choosing appropriate environments and husbandry methods for captive animals.

Environmental enrichment is the addition of stimuli (beyond minimal food and shelter needed for survival) to a captive animal's environment with the objective of improving the animal's well being. Enrichment can be used to improve animal welfare, encourage successful reproduction, and can potentially reduce the occurrences and manifestations of chronic stress (Mellen \& MacPhee, 2001). Several researchers have focused on enrichment as a means to increase species-specific behaviors (Markowitz \& Lafrose, 1987; Mellen \& MacPhee; 2001; Renner \& Lussier, 2002) and decrease stereotypic behaviors (Mason, 1991; Mellen \& MacPhee, 2001).

A nonhuman animal and carnivore, the coyote (Canis latrans) is a canid who inhabits much of North America (Bekoff \& Gese, 2003) and has been studied intensively due to the animal's economic importance as a predator of livestock (Knowlton, Gese, \& Jaeger, 1999). Thus, the coyote is an important species to study under controlled conditions of captivity. However, as a wide-ranging animal, there could be complications involved with maintaining coyotes in captivity (Clubb \& Mason, 2003). Similarly, inferences made from research on captive coyotes should account for potential differences between 
captive and wild behavioral repertoires before being applied in management situations.

Published literature insufficiently examines the effects of captivity and enrichment on coyote behavior. Thus, the objective of our research was to measure how behavioral budgets of captive coyotes differed from wild coyotes and if environmental enrichment could reduce stereotypical behaviors and stress in captive coyotes. This study used an interdisciplinary approach (Lund, Coleman, Gunnarsson, Appleby, \& Karinen, 2006) and attempted to measure welfare in the context of natural history and physiology. Specifically, it examined the effect of (a) environment (wild or captive enclosures) on behavioral budget and stereotypic behavior and (b) environmental enrichment on behavioral budget, stereotypic behavior, and corticosteroid levels.

\section{METHOD}

\section{Subjects and Study Sites}

Eight adult ( $>2$ years of age) captive coyotes ( 4 males, 4 females) were subjects for the trials during July-September 2005. All coyotes were born, raised, and housed at the United States Department of Agriculture Wildlife Services National Wildlife Research Center's Predator Research Facility in Millville, Utah. Research activities were reviewed and approved by the center's Institutional Animal Care and Use Committee (QA 1285).

Two types of enclosures were used: large outdoor enclosures and holding kennels and 4 coyotes ( 2 males and 2 females) were randomly assigned to either enclosure type for the duration of the study. Each outdoor enclosure consisted of a teardrop-shaped $1,000 \mathrm{~m}^{2}$ fenced area (Figure 1). Inside each enclosure were two raised platforms $(1.2 \mathrm{~m} \times 0.9 \mathrm{~m} \times 0.6 \mathrm{~m}$ shade shelters). Each enclosure abutted a small observation building that also contained a den box $(60 \mathrm{~cm}$ diameter plastic cylinders that coyotes "could enter ad libitum). Each outdoor enclosure housed a mated pair of coyotes. Holding kennels were $2.5 \mathrm{~m} \times 1.2 \mathrm{~m}$ enclosures, with chain-link walls. Floors were rubberized grating suspended 30 $\mathrm{cm}$ above a concrete floor. Inside each kennel, coyotes had access to a raised platform measuring $60 \mathrm{~cm} \times 45 \mathrm{~cm}$ and a den box (of the same design used in the outdoor pens) that opened into the kennel. A $60^{\prime} \mathrm{cm}$ tall visual barrier was installed between adjacent kennels. Although the kennel complex was covered by a roof, it was open to the environment on all four sides. Coyotes were housed individually in each kennel but were directly adjacent to their historical mate (as those paired in pens).

Because coyotes are assigned to housing, tracked, and cared for as individuals, the individual coyote was considered the sample unit for analysis. Thus, sample 


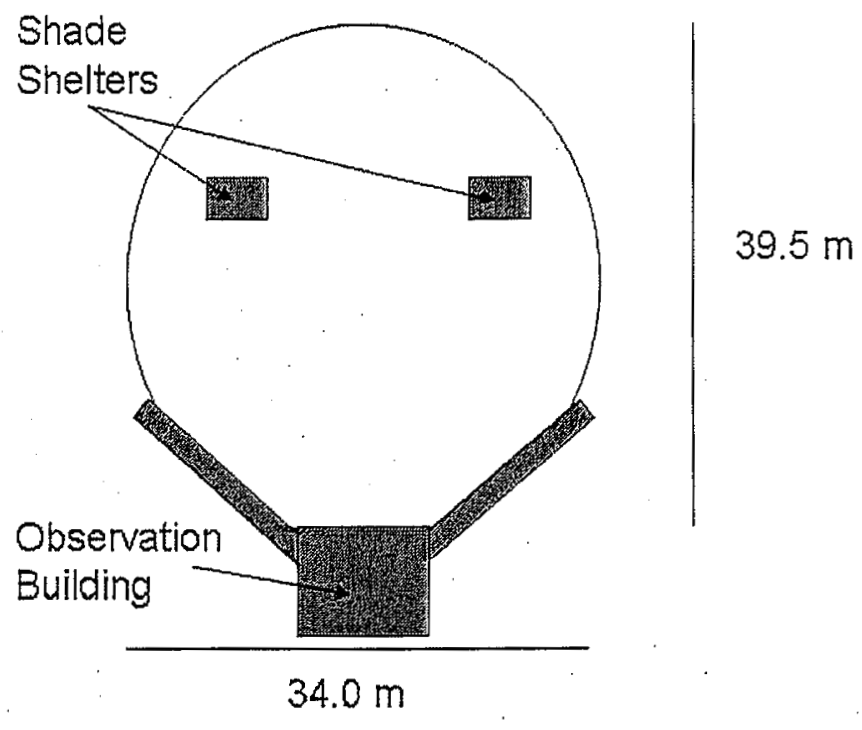

FIGURE 1 Diagram of the outdoor pen design at the Millville Predator Research Facility.

size for coyotes in the kennels was 4 (although were all housed in the same kennel block), and sample size for coyotes in pens was also 4 (although they were paired in two separate pens).

The wild population used for comparison was observed by Gese (1995) in Yellowstone National Park. Data from 8 coyotes ( 6 male and 2 female) were sampled from observations made during April and August 1991-1993 (Gese, Ruff, \& Crabtree, 1996). Animals were observed under natural conditions in the Lamar Valley of northern Yellowstone; animals were free to roam, although at the beginning of the study they were handled in order to fit them with radio. collars.

\section{Data Collection and Observations}

In captivity, each subject was observed for $1 \mathrm{hr}$ each day for 16 days using focal sampling (16 hr per coyote, total $128 \mathrm{hr}$ ). Observations took place between 0530 and 1000 , and between 1600 and 2100; observations of each animal were balanced to include equal numbers of morning and evening observations throughout the study. Observations of captive coyotes were recorded directly on a laptop computer using Observer (Version 5, Noldus Information Technology) software. Behaviors recorded were based on the ethogram (Table 1) used by Gilbert-Norton (2004), as adapted from Gese et al. (1996).

Observations of wild coyotes were also performed using focal animal sampling and a microcomputer with custom software (Gese, 1995). Time budgets 
TABLE 1

Ethogram of Behaviors Used for Comparison of Wild and Captive Coyotes

\begin{tabular}{|c|c|}
\hline Behavior & Description \\
\hline Resting & Coyote laying on side with head up or down or sitting on its haunches. \\
\hline Locomotion & Purposeful walking, trotting, or running using whole body movement with head up. \\
\hline Standing & Standing still with head raised. \\
\hline Foraging & Orientating, stalking, and searching at slow pace with head lowered. \\
\hline Eating & Coyote eating (visible jaw movement). \\
\hline Social & $\begin{array}{l}\text { Directed action toward another individual, e.g., directed aggression or licking, } \\
\text { biting, play bounding, chasing. }\end{array}$ \\
\hline Stereotyping & $\begin{array}{l}\text { A repetitive movement (with no apparent goal or function) during which the } \\
\text { coyote repeats the exact movement for greater than } 2 \text { cycles. }\end{array}$ \\
\hline
\end{tabular}

of wild coyotes were created using $150.5 \mathrm{hr}$ of observations $(\bar{x}=18.8 \mathrm{hr}$ per coyote) matched to season and time of day of the captive study.

All data were classified into the proportion of time coyotes spent exhibiting the different behaviors. For the initial analysis, data were classified to allow direct comparison between wild and captive animals. That is, in the captive data set, stereotyping and traveling behaviors were pooled as locomotion for comparison to wild coyotes because pacing is an active behavior and can be considered a form of locomotion regardless of whether it is considered abnormal.

\section{Comparison of Behaviors Between Captive and Wild Environments}

This study compared behavioral budgets, the proportion of time active coyotes (in kennels, pens, or in the wild) spent in each behavioral category (Table 1). Housing type included differences in husbandry, location on the site or valley, substrate, and a variety of other factors; thus, housing type is a treatment that describes more than the size of the area the coyotes occupied. Individual coyotes were randomly assigned to kennel or pen, and wild animals were randomly selected from those under observation; thus, behavioral budgets between animals were compared using Kruskal-Wallis test statistic ( $H$, SYSTAT Version 5.04). Nonparametric multiple comparisons (Nemenyi procedure, Q, Zar, 1984) were used for comparisons between housing types when Kruskal-Wallis test results indicated significant differences.

The study also included an analysis that compared the effect of housing type (kennel, pen, or wild) on the display of stereotypic behavior. Differences in the proportion of time that coyotes spent stereotyping were assessed using a Kruskal-Wallis test. If differences were detected, the Nemenyi procedure was used for multiple comparisons. 


\section{Effect of Environmental Enrichment on Behavior and Corticosteroid Levels}

To examine the effect of environmental enrichment on behavioral budgets of captive coyotes, a baseline-experimental condition-baseline (ABA structure) repeated measures design was used to examine behavioral budgets before, during, and after enrichment was provided.

The object used for environmental enrichment was a sterile compressed hollow bone (Petsmart ${ }^{\mathrm{TM}} \mathrm{X}$-large natural bone). Bones were provided either empty or filled with food. In the food-filled bone, green lettuce leaves were placed in one end, then the bone was filled with a teaspoon of enriched liver paste (Kong Stuff' $\mathrm{n}^{\circledR}$ ) and six live adult crickets (Euscyrtus concinnus) and sealed with another set of green lettuce leaves. Bones that were left empty were handled for the same duration of time as bones that were filled with food, and all bones were handled using latex gloves to minimize the amount of human scent transferred to the bones.

Presentation of the bone stimulus varied during the enrichment.period; on the 1st day, a bone was provided (or two identical bones in the enclosures that held pairs of coyotes). Bones were randomly chosen to be filled or not filled on initial presentation. The bones were left in the enclosure until the end of the 2nd day and then the alternate form (filled or not filled) was added to the enclosure before the first observation on the 3rd day. All bones were then removed after the evening observation on the 4 th day. This pattern of enrichment was repeated for the next 4 days to complete the enrichment period.

Therefore, there was a 4-day baseline period (preenrichment), then an 8day enrichment period, and finally a 4-day postenrichment period. Behavioral budgets were analyzed using Friedman's tests $\left(X_{\text {ra,b) }}^{2}\right.$ and the Nemenyi procedure where appropriate.

The study also examined the effect of environmental enrichment on coyote stereotypic behavior when enrichment bones were present versus the pre- and postenrichment periods. In kennels and pens, the ABA repeated measures design described earlier was again employed and differences identified using Friedman's tests and the Nemenyi procedure when appropriate.

To measure corticosteroid levels for stress analyses, researchers collected scats that were deposited by each of the 4 coyotes housed in kennels during preenrichment, enrichment, and postenrichment periods. Collected scats were scraped from kennel floors every morning after observations were made. All scats in each.kennel were combined into one mixed sample in order to minimize effects of time of day on corticosteroid level; that is, corticosteroids were sampled and analyzed as mean daily values. Scats were stored in labeled plastic freezer bags and immediately frozen $\left(-80^{\circ} \mathrm{C}\right)$. Scats were not collected from outdoor enclosures because coyotes there were paired and individual identification of the scat's depositor was not possible. 
For lab analyses, approximately $0.5 \mathrm{~g}$ of fecal material from each scat was shaken overnight in $5 \mathrm{~mL}$ modified phosphate-saline buffer containing 50\% methanol, $0.1 \%$ bovine serum albumin, and 5\% Tween 20 (Shiedler, Ortuño, Morán, Moorman, \& Lasley, 1993). Fecal corticoid levels were determined by radioimmunoassay. Antibody-coated tubes and 125-I cortisol (Active ${ }^{\mathbb{1}}$ Cortisol RIA) were obtained from Diagnostic Systems Laboratory (DSL, Webster, TX). Reference standard cortisol (Sigma-Aldrich, St. Louis, MO) was diluted in phosphate-buffered saline (PBS), pH 7.0. Assays were performed according to manufacturer's protocols with the exception that the matrices were equalized by adding PBS to the fecal samples and fecal extraction buffer (containing 50\% methanol) to the standards.

Concentrations were determined as $\mathrm{ng} / \mathrm{mL}$ and then divided by the dry weight of feces extracted to give the results as ng/g feces. All samples were assayed in duplicate. The ABA repeated measures design described previously was again used for corticosteroid analyses. Corticosteroid levels were compared before, during, and after environmental enrichment using Friedman's tests and the Nemenyi procedure. Because variability of corticosteroid levels in response to enrichment can also be an important metric, we also assessed for differences in variance between preenrichment, enrichment, and postenrichment periods using a Bartlett's test (Zar, 1984).

\section{RESULTS}

\section{Comparison of Behaviors Between Captive and Wild Environments}

Overall, behavioral repertoires of wild coyotes were similar to those of coyotes in captivity, but captive coyote behavior diverged from wild behavior in only two behavioral categories: standing and foraging (Table 2). Specifically, kenneled coyotes stood much more than wild coyotes $(Q=3.04, p<.01)$, but the proportion of time standing was similar between wild coyotes and penned coyotes $(Q=1.16, p>.50)$ and penned coyotes and kenneled coyotes $(Q=$ 1.63, $p>.50)$. Wild coyotes foraged more than coyotes in pens $(Q=2.52$, $p<.05)$, but the proportion of time spent foraging was similar for wild and kenneled coyotes $(Q=1.07, p>.50)$ and kenneled and penned coyotes $(Q=$ $1.26, p>.50)$.

Coyotes in the various housing types showed different amounts of stereotyping ( $H=12.99, p=.02)$; the proportion of time spent stereotyping in kennels $(\bar{x}=0.23)$ was not statistically different from that spent by coyotes in the pens $(\bar{x}=0.112, Q=0.30, p>.5)$. Coyotes in the wild did not stereotype at all, which was statistically different from what was exhibited by coyotes in the pens $(Q=3.77, p<.001)$ and the kennels $(Q=4.12, p<.001)$. 
TABLE 2

Mean Proportion of Time Active Coyotes Spent

Exhibiting Different Behaviors by Housing Type

\begin{tabular}{lllll}
\hline & \multicolumn{4}{c}{ Housing Type } \\
\cline { 2 - 5 } Behavior & Wild $(90 \%$ C.I.) & Kennel (90\% C.I.) & Pen $(90 \%$ C.I. $)$ & $p$ \\
\hline Resting. & $0.58(0.39-0.78)$ & $0.55(0.47-0.64)$ & $0.58(0.55-0.61)$ & .76 \\
Locomotion & $0.19(0.09-0.29)$ & $0.15(0.05-0.24)$ & $0.21(0.17-0.26)$ & .66 \\
Standing & $0.12(0.06-0.18)$ & $0.23(0.18-0.27)$ & $0.16(0.11-0.20)$ & .01 \\
Foraging & $0.09(0.04-0.13)$ & $0.06(0.02-0.09)$ & $0.03(0.01-0.04)$ & .04 \\
Eating & $0.01(-0.01-0.04)$ & $0.00(0.00-0.00)$ & $0.01(0.00-0.02)$ & .12 \\
Social & $0.01(-0.01-0.03)$ & $0.01(0.00-0.02)$ & $0.02(0.00-0.04)$ & .59 \\
\hline
\end{tabular}

Note. C.I. $=$ confidence interval.

Effect of Environmental Enrichment on Behavior and Corticosteroid Levels

Coyotes in the kennels (Table 3 ) were affected by enrichment because resting behavior was observed nearly half as much during the enrichment period compared to preenrichment $(Q=3.31, p<.005$ ); the proportion of time resting postenrichment was similar to that in the preenrichment period $(Q=0.42, p>$ .50 ), but coyotes also rested more after the enrichment period than during it ( $Q=$ $2.91, p<.02)$. Also, there was far more foraging behavior observed during the enrichment period than postenrichment $(Q=3.74, p<.005)$, although there were no statistically significant differences between preenrichment and

TABLE 3

Mean Proportion of Time Active Coyotes in Kennels Spent

Exhibiting Different Behaviors by Testing Period

\begin{tabular}{llllll}
\hline & \multicolumn{5}{c}{ Enrichment Period } \\
\cline { 2 - 5 } Behavior & Pre $(90 \%$ C.I. $)$ & During $(90 \%$ C.I. $)$ & Post $(90 \%$ C.I. $)$ & $p$ \\
\hline Resting & $0.55(0.47-0.64)$ & $0.27(0.13-0.40)$ & $0.57(0.35-0.80)$ & .04 \\
Locomotion & $0.15(0.05-0.24)$ & $0.25(0.00-0.50)$ & $0.23(-0.01-0.47)$ & .37 \\
Standing & $0.23(0.18-0.27)$ & $0.16(0.08-0.24)$ & $0.15(0.08-0.22)$ & .48 \\
Foraging & $0.06(0.02-0.09)$ & $0.30(0.05-0.54)$ & $0.03(0.00-0.05)$ & .02 \\
Eating & $0.00(0.00-0.00)$ & $0.02(0.01-0.04)$ & $0.01(-0.01-0.04)$ & .07 \\
Social & $0.01(0.00-0.02)$ & $0.00(0.00-0.00)$ & $0.01 \cdot(0.00-0.02)$ & .27 \\
\hline
\end{tabular}

Note. C.I. = confidence interval. 
enrichment $(Q=1.66, p>.20$ ) or preenrichment and postenrichment $(Q=$ 2.08, $p>.20$ ).

Behavioral repertoires of penned coyotes were also similar before, during, and after enrichment was provided (Table 4), with the exception of resting, locomotion, and foraging behaviors. Coyotes rested less during the enrichment period than postenrichment $(Q=3.60, p<.001)$, but preenrichment resting was similar to the enrichment period $(Q=1.39, p>.20)$ and the postenrichment period ( $Q=2.22, p>.05$ ). Coyotes were much more active, in terms of locomotive behavior, during enrichment than after $(Q=4.30, p<.001)$, but the amount of locomotive behavior was similar in the pre- and enrichment conditions $(Q=1.94, p>.10)$ and the pre- and postenrichment conditions $(Q=2.36$, $p>.05$ ). Similarly, coyotes foraged more during the enrichment period than afterward $(Q=3.61, p<.001)$, but the amount of foraging was similar before and during the enrichment period $(Q=2.08, p>.10)$ and the before and after periods $(Q=1.53, p>.20)$.

For coyotes in kennels, the proportion of time spent doing stereotypic behavior was not significantly different between preenrichment $(\bar{x}=0.15)$, enrichment $(\bar{x}=0.25)$, and postenrichment $(\bar{x}=0.29)$ periods $\left(X_{\mathrm{r} 3,4}^{2}=3.5, p=.17\right)$. Coyotes in pens differed in their proportion of time spent exhibiting stereotypic behaviors during different enrichment periods $\left(X_{\mathrm{r} 3,4}^{2}=8.00, p=.02\right)$. Post hoc comparisons indicated that there was no statistically significant difference between the enrichment period $(\bar{x}=0.12)$ and the pre- $(\bar{x}=0.20, Q=$ $0.83, p>.50$ ) or postenrichment period $(\bar{x}=0.02, Q=1.66, p>.2)$. The postenrichment period was significantly less than the preenrichment period, however $(Q=2.50, p<.05)$.

For coyotes in kennels, there was little evidence that corticosteroid levels (ng/g feces) were altered by environmental enrichment during preenrichment

TABLE 4

Mean Proportion of Time Active Coyotes in Outdoor Pens Spent Exhibiting Different Behaviors by Testing Period

\begin{tabular}{lllll}
\hline & & \multicolumn{4}{c}{ Enrichnent Period } \\
\cline { 2 - 5 } Behavior & Pre $(90 \%$ C.I.) & During $(90 \%$ C.I. $)$ & Post $(90 \%$ C.I. $)$ & $p$ \\
\hline Resting & $0.58(0.55-0.61)$ & $0.39(0.15-0.63)$ & $0.70(0.60-0.80)$ & .02 \\
Locomotion & $0.21(0.17-0.26)$ & $0.32(0.20-0.44)$ & $0.11(0.08-0.13)$ & .02 \\
Standing & $0.16(0.11-0.20)$ & $0.21(0.12-0.30)$ & $0.14(0.09-0.20)$ & .11 \\
Foraging & $0.03(0.01-0.04)$ & $0.05(0.02-0.08)$ & $0.02(0.01-0.03)$ & .02 \\
Eating & $0.01(0.00-0.02)$ & $0.02(-0.01-0.04)$ & $0.00(0.00-0.0)$ & .83 \\
Social & $0.02(0.00-0.04)$ & $0.01(0.00-0.03)$ & $0.02(-0.01-0.06)$ & .99 \\
\hline
\end{tabular}

Note. C.I. $=$ confidence interval. 
$(\bar{x}=74.20, S E=4.06)$, enrichment $(\bar{x}=84.25, S E=10.62)$, or postentichment $(\bar{x}=75.43, S E=7.61)$ periods $\left(X_{13,4}^{2}=2.0, p>.20\right)$; nor did we detect a significant difference in variability of responses from coyotes $(p=.37)$.

\section{DISCUSSION}

Holding medium-size carnivores in captivity and minimizing the area in which they can travel is expected to have impacts on behavior (Clubb \& Mason, 2003). In this work, however, although housing type did appear to influence the behavioral budgets of coyotes, the effects were not drastic and apparent across all behavioral categories. Why coyotes were observed standing less in the wild than in kennels is unclear; it may be that captive coyotes need to stand to observe areas outside of small, roofed, and visually restricted kennels.

To align proportions of standing behaviors with those observed in the wild, it may be useful to have larger pens or visually unobstructed views for captive coyotes, and such an experiment should be considered. Differences in foraging behaviors between wild coyotes and those in pens could possibly be minimized by altering food provisioning schedules. Size was not the only difference between housing types, and differences in behaviors could be due to differences in substrate, husbandry techniques, proximity to other animals, or many other possibilities. Thus, other factors should be considered when designing or assessing captive environments for carnivores such as coyotes.

Stereotypic behaviors were not observed in the wild, as expected, but they were apparent in both kennels and pens. This study did not measure when coyotes developed their particular stereotypic behaviors, and some coyotes could have developed them at different times during their history in the colony. That is, coyotes could have developed abnormal behaviors while previously in pens or kennels. Although housing may contribute to the development of stereotypic behavior, this work provides little evidence that something as simple as altering housing type will eliminate stereotypic behaviors.

Certain behaviors, such as searching or foraging, may be relatively easy to increase with enrichment (Ings, Waran, \& Young, 1997). Indeed, captive coyotes responded to the enrichment stimuli we provided, as inferred from an increase in foraging behavior. However, showing a response to enriching stimuli does not necessarily mean an improvement in stress levels or behavioral repertoires; more of any particular behavior does not necessarily indicate better housing condition or welfare. Enrichment increased activity and influenced behavior but did not have as large of (if any) effect for altering stress hormones or promoting more natural behavioral budgets.

In neither kennels nor pens was the enrichment period associated with the least amount of stereotyping; although no significant differences between pe- 
riods were observed in kennels, coyotes in pens exhibited the least amount of stereotyping after enriching stimuli were removed. Intrinsic and environmental factors can contribute to stereotyping or other negative behaviors (Lutz, Well, \& Novak, 2003); in addition, in some species, environmental enrichment can influence the development of stereotypic behaviors (Caston et al., 199.9, Powell, Newman, McDonald, Bugenhagen, \& Lewis, 2000).

Relative to stress and corticosteroid levels, we were only able to measure corticosteroid levels in captive animals, and we did not have a baseline of what levels should be (in reference to a wild population). Thus, we cannot infer what the measured corticosteroid levels ultimately indicate, only that, on average, they were not drastically affected by enrichment. Another important limitation of our experiment was our small sample size; although we did not detect a statistical difference in variability between enrichment periods, the doubling of the standard errors after enrichment gave us reason to believe that enrichment can affect corticosteroid levels in some animals. Future experiments, with greater statistical power, would be useful.

Of interest to facilities that support colonies of captive carnivores is the behaviors of our captive coyotes; when defined in broad categories, their behavioral budgets were very similar to those of wild coyotes. This suggests that captivity does not necessarily mean poor welfare or scientific inference due to inability to perform species-typical behaviors. To conclude, however, that behavioral budgets of captive animals are equivalent to those of their wild counterparts relies heavily on the classification of stereotypic behavior. Coyotes spend much of their active time traveling (Gese et al., 1996); therefore, it is possible that stereotypic pacing could be a consequence of fulfilling a biological motivation to travel or move.

It can be argued that, if captive coyotes are traveling (defining stereotypic movements as traveling) for the same period of time as wild counterparts, then stereotyping is an innocuous adaptation to captivity. However; it is clear that stereotyping is an abnormal behavior (Mason \& Latham, 2004; Vestergaard, Skadhauge, \& Lawson, .1997) because repetitive pacing in a $1 \mathrm{~m}$ area is not observed in the wild. Holding coyotes in small environments may result in more stereotyping; unfortunately, as reported in other studies, the introduction of simple enriching stimuli or larger pens is likely not an immediate panacea for behavioral abnormalities (Vinke, Houx, Van Den Bos, \& Spruijt, 2005).. Continued, long-term studies in larger pens would be useful.

\section{CONCLUSION}

In summary, the strength and relevance of this study is threefold. First, coyotes in captivity, when examined using broad behavioral categories, acted similarly to 
coyotes in the wild; however, some behaviors, especially foraging, were sensitive to different housing or enrichment conditions.

Second, captive coyotes showed some abnormalities in behavior (in the form of stereotypic behaviors that were not observed in the wild), and the introduction of a simple enrichment object or different housing conditions did not immediately correct these abnormalities.

Third, a physiological indicator of stress was not reduced upon introduction of a simple enriching stimulus. Conclusions should be interpreted acknowledging limited sample sizes ( 4 coyotes per treatment), but the importance of any experimental information for the welfare of captive carnivores should not be discounted.

\section{ACKNOWLEDGMENT}

This study was funded by the National Wildlife Research Center. Research and Institutional Animal Care and Use protocols were approved by the National Wildlife Research Center (QA 1285). We are grateful to the staff and volunteers at the Wildlife Services National Wildlife Research Center's Predator Research Facility in Millville, UT, for their time, effort, help, and patience during the study. We especially thank Stacey Brummer for sharing her knowledge and Lynne Gilbert-Norton for her support and assistance. We also thank Joan Bauman and personnel at the Saint Louis Zoo for the analyses of fecal corticosteroid levels.

\section{REFERENCES}

Becker, B. A., Nienaber, J. A., Christenson, R. K., Manak, R. C., DeShazer, J. A., \& Hahn, G. L. (1985). Peripheral concentrations of cortisol as an indicator of stress, in the pig. American, Joumal. Veterinary, Research, 46, 1034-1038.

Bekoff, M., \& Gese, E. M. (2003). Coyote (canis latrans). In G: A. Feldhammer, B. C. Thompson, \& A. Chapman (Eds.), Wild mammals of North America: Biology, management and conservation (pp. 467-481). Baltimore: Johns Hopkinis University Press.

Carlstead, K., Seidensticker, J., \& Baldwin, R. (1991). Environmental enrichment for zoo bears. Zoo Biology. 10, 3-16.

Caston, J., Devulder, B., Jouen, F., Lalonde, R., Delhaye-Bouchaud, N., \& Mariani, J. (1999). Role of an enriched environment on the restoration of behavioral deficits in Lurcher mutant mice. Developmental Psychobiology, 3.5, 291-303.

Clubb, R., \& Mason, G. (2003). Captivity effects on wide-ranging carnivores. Nature, 425, 473-474.

Dantzer, R. (1986). Behavioral, physiological and functional aspects of stereotyped behavior: A review and a re-interpretation. Journal of Animal Science, 62, 1776-1786.

Garner, J. P., Mason, G. J., \& Smith, R. (2003). Stereotypic route-tracing in experimentally caged songbirds correlates with general behavior disinhibition. Animal Behaviour; 66, 237-249.

Gese, E. M. (1995). Foraging ecology' of coyotes in Yellowstone National Park. Unpublished doctoral dissertation, University of Wisconsin, Madison. 
Gese, E. M., Ruff., R. L., \& Crabtree, R. L. (1996). Foraging ecology' of coyotes (canis latrans): The influence of extrinsic factors and a dominance hierarchy. Canadian Joumal of Zoology, 74, $769-783$.

Gilbert-Norton, L. (2004). The predictability of food resources, and its effect on foraging and exploratory behaviour of captive coyotes (Canis latrans). Unpublished master's thesis, University of Exeter, Exeter, UK.

Herbers, J. M. (1981). Time resources and laziness in animals. Oecologia, 49, 252-262.

Ings, R., Waran, N. K., \& Young, R. J. (1997). Effect of wood-pile feeders on the behaviour of captive bush dogs (Speothos venaticus). Animal Welfare, 6, 145-152.

Knowlton, F. F., Gese, E. M., \& Jaeger, M. M. (1999). Coyote depredation control: An interface between biology and management. Journal of Range Management, 52, 398-412.

Lund, V., Coleman, G., Gunnarsson, S., Appleby, M. C., \& Karinen, K. (2006). Animal welfare science: Working at the interface between the natural and social sciences. Applied Animal Behaviour Science, 97, 37-49.

Lutz, C., Well, A., \& Novak, M. (2003). Stereotypic and self-injurious behavior in thesus macaques: A survey and retrospective analysis of environment and early experience. American Journal of Primatology, 60, 1-15.

Markowitz, H., \& LaFrose, S. (1987). Artificial prey as behavioural enrichment devices for felines. Applied Animal Behaviour Science, 18, 31-43.

Mason, G. J. (1991). Stereotypics: A critical review. Animal Behavioul; 41, 1015-1037.

Mason, G. J., \& Latham, N. R. (2004). Can't stop, won't stop: Is stereotyping a reliable animal welfare indicator? Animal Welfare, 13, 57-69.

Mellen, J. D., \& MacPhee, M. S. (2001). Philosophy of environmental enrichment: Past, present and future. Zoo Biology, 20, 211-266.

Morton, D. J., Anderson, E., Foggin, C. M., Kock, M. D., \& Tiran, E. P. (1995). Plasma cortisol. as an indicator of stress due to capture and translocation in wildlife species. Veterinary Record, $136,60-63$.

Newberry, R. C. (1995). Environmental enrichment: Increasing the biological relevance of captive environments. Applied Animal Behaviour Science, 44, 229-243.

Powell, S. B., Newman, H. A., McDonald, T. A., Bugenhagen, P., \& Lewis, M. H. (2000). Development of spontaneous stereotyped behavior in deer mice: Effects of early and late exposure to a more complex environment. Developmental. Psychobiology, 37, 100-108.

Renner, M. J., \& Lussier, J. P. (2002). Environmental enrichment for captive spectacled bear (Tremarctos omatus). Pharmacology Biochemistry' and Behavior; 73, 279-283.

Shepherdson, D. J., Carlstead, K., Mellen, J. D., \& Sidensticker, J. (1993). The influence of food presentation on the behavior of small cats in confined environments. Zoo Biology, 12; 203-216.

Shiedler, S. E., Ortuño, A. M., Morán, F. M., Moorman, E. A., \& Lasley, B. L. (1993). Simple extraction and enzyme immunoassays for estrogen and progesterone metabolites in the feces of Macaca fascicularis during non-conceptive and conceptive ovarian cycles. Biology of Reproduction, 48 , 1290-1298.

Spinka, $\dot{M}$. (2006). How important is natural behaviour in animal farming systems? Applied Animal Beliaviour Science, 100, 117-128.

Terlouw, E. M. C., Lawrence, A. B., \& Illius, A. (1991). Influences of feeding level and physical restriction on the development of stereotypies in sows. Animal Behaviour; 42, 981-991.

Veasey, J. S., Waran, N. K., \& Young, R. J. (1996). On comparing the behaviour of zoo housed animals with wild conspecifics as a welfare indicator. Animal Welfare, 5, 13-24.

Vestergaard, K. S., Skadhauge, E., \& Lawson, L. G. (1997). The stress of not being able to perform dust bathing in laying hens, Physiology and Behavior, 62, 413-419.

Vinke, C. M., Houx, B. B., Van Den Bos, R., \& Spruijt, B. M. (2005). Anticipatory behaviour and stereotypical behaviour in farmed mink (Mustela vison) in the presence, absence and after the removal of swimming water. Applied Animal Behaviour Science, 96, 129-142.

Zar, J. H. (1984). Biostatistical analysis (2nd ed.). Saddle River, NJ: Prentice Hall. 